ARTICLE

Received 2 Dec 2013 | Accepted 2 Apr 2014 | Published 7 May $2014 \quad$ DOl: 10.1038/ncomms4786

\title{
Observation of a three-dimensional topological Dirac semimetal phase in high-mobility $\mathrm{Cd}_{3} \mathrm{As}_{2}$
}

\author{
Madhab Neupane ${ }^{1, \star}$, Su-Yang $X u^{1, \star}$, Raman Sankar ${ }^{2, \star}$, Nasser Alidoust ${ }^{1}$, Guang Bian ${ }^{1}$, Chang Liu', Ilya Belopolski ${ }^{1}$, \\ Tay-Rong Chang ${ }^{3}$, Horng-Tay Jeng ${ }^{3,4}$, Hsin Lin $^{5}$, Arun Bansil ${ }^{6}$, Fangcheng Chou ${ }^{2} \&$ M. Zahid Hasan ${ }^{1,7}$
}

Symmetry-broken three-dimensional (3D) topological Dirac semimetal systems with strong spin-orbit coupling can host many exotic Hall-like phenomena and Weyl fermion quantum transport. Here, using high-resolution angle-resolved photoemission spectroscopy, we performed systematic electronic structure studies on $\mathrm{Cd}_{3} \mathrm{As}_{2}$, which has been predicted to be the parent material, from which many unusual topological phases can be derived. We observe a highly linear bulk band crossing to form a 3D dispersive Dirac cone projected at the Brillouin zone centre by studying the (001)-cleaved surface. Remarkably, an unusually high in-plane Fermi velocity up to $1.5 \times 10^{6} \mathrm{~ms}^{-1}$ is observed in our samples, where the mobility is known up to $40,000 \mathrm{~cm}^{2} \mathrm{~V}^{-1} \mathrm{~s}^{-1}$, suggesting that $\mathrm{Cd}_{3} \mathrm{As}_{2}$ can be a promising candidate as an anisotropic-hypercone (three-dimensional) high spin-orbit analogue of 3D graphene. Our discovery of the Dirac-like bulk topological semimetal phase in $\mathrm{Cd}_{3} \mathrm{As}_{2}$ opens the door for exploring higher dimensional spin-orbit Dirac physics in a real material.

\footnotetext{
${ }^{1}$ Joseph Henry Laboratory, Department of Physics, Princeton University, Princeton, New Jersey 08544, USA. ${ }^{2}$ Center for Condensed Matter Sciences, National Taiwan University, Taipei 10617, Taiwan. ${ }^{3}$ Department of Physics, National Tsing Hua University, Hsinchu 30013, Taiwan. ${ }^{4}$ Institute of Physics, Academia Sinica, Taipei 11529, Taiwan. ${ }^{5}$ Graphene Research Centre, Department of Physics, National University of Singapore, Singapore 117542 , Singapore. ${ }^{6}$ Department of Physics, Northeastern University, Boston, Massachusetts 02115, USA. 7 Princeton Center for Complex Materials, Princeton University, Princeton, New Jersey 08544, USA. * These authors contributed equally to this work. Correspondence and requests for materials should be addressed to M.Z.H. (email: mzhasan@Princeton.edu).
} 
T wo-dimensional (2D) Dirac electron systems exhibiting many exotic quantum phenomena constitute one of the most active topics in condensed matter physics ${ }^{1-19}$. The notable examples are graphene and the surface states of topological insulators (TIs). Three-dimensional (3D) Dirac fermion metals, sometimes noted as the topological bulk Dirac semimetal (BDS) phases, are also of great interest if the material possesses $3 \mathrm{D}$ isotropic or anisotropic relativistic dispersion in the presence of strong spin-orbit coupling. It has been theoretically predicted that a topological (spin-orbit) 3D spin-orbit Dirac semimetal can be viewed as a composite of two sets of Weyl fermions where broken time-reversal or space inversion symmetry can lead to a surface Fermi-arc semimetal phase or a $\mathrm{TI}^{14}$. In the absence of spin-orbit coupling, topological phases cannot be derived from a 3D Dirac semimetal. Thus, the parent BDS phase with strong spin-orbit coupling is of great interest. Despite their predicted existence ${ }^{11,13,14}$, experimental studies on the massless BDS phase have been lacking as it has been difficult to realize this phase in real materials, especially in stoichiometric single crystalline non-metastable system with high mobility. It has also been noted that the BDS state can be achieved at the critical point of a topological phase transition ${ }^{20,21}$ between a normal insulator and a TI, which requires fine-tuning of the chemical doping/alloying composition thus by effectively varying the spin-orbit coupling strength. This approach also introduces chemical disorder into the system. In stoichiometric bulk materials, the known 3D Dirac fermions in bismuth are in fact of massive variety as there clearly exists a band gap in the bulk Dirac spectrum ${ }^{10}$. On the other hand, the bulk Dirac fermions in the $\mathrm{Bi}_{1-x} \mathrm{Sb}_{x}$ system coexist with additional Fermi surfaces ${ }^{5}$. Therefore, to this date, identification of a gapless BDS phase in stoichiometric materials remains experimentally elusive.

In this Article, we present the experimental identification of a gapless Dirac-like 3D topological (spin-orbit) semimetal phase in stoichiometric single crystalline system of $\mathrm{Cd}_{3} \mathrm{As}_{2}$, which is protected by the $C_{4}$ crystalline (crystal structure) symmetry and spin-orbit coupling as predicted in theory ${ }^{14}$. Using highresolution angle-resolved photoemission spectroscopy (ARPES), we show that $\mathrm{Cd}_{3} \mathrm{As}_{2}$ features a bulk band Dirac-like cone locating at the centre of the (001) surface projected Brillouin zone (BZ). Remarkably, we observe that the band velocity of the bulk Dirac spectrum is as high as $\sim 10 \mathrm{eV} \AA$, which along with its massless character favourably contributes to its natural high mobility $\left(\sim 10^{5} \mathrm{~cm}^{2} \mathrm{~V}^{-1} \mathrm{~s}^{-1}\right.$ (refs 22,23$\left.)\right)$. We further compare and contrast the observed crystalline-symmetry-protected BDS phase in $\mathrm{Cd}_{3} \mathrm{As}_{2}$ with those of in the Bi-based 3D-TI systems such as in $\mathrm{BiTl}\left(\mathrm{S}_{1-\delta} \mathrm{Se}_{\delta}\right)_{2}$ and $\left(\mathrm{Bi}_{1-\delta} \mathrm{In}_{\delta}\right)_{2} \mathrm{Se}_{3}$ systems. Our experimental identification and band-structure measurements of the Dirac-like bulk semimetal phase and its clear contrast with $\mathrm{Bi}_{2} \mathrm{Se}_{3}$ and $2 \mathrm{D}$ graphene discovered previously, opens the door for exploring higher dimensional spin-orbit Dirac physics in a stoichiometric material. These new directions are uniquely enabled by our observation of strongly spin-orbit coupled 3D massless Dirac semimetal phase protected by the $C_{4}$ symmetry, which is not possible in the 2D Dirac fermions in graphene and the surfaces of TIs, or weak spin-orbit 3D Dirac fermions in other materials.

\section{Results}

Crystalline symmetry protected topological Dirac phase. The crystal structure of $\mathrm{Cd}_{3} \mathrm{As}_{2}$ has a tetragonal unit cell with $a=12.67 \AA$; and $c=25.48 \AA$; for $Z=32$ with symmetry of space group $I 4_{1} \mathrm{~cd}$ (see Fig. 1a,b). In this structure, arsenic ions are approximately cubic close-packed and Cd ions are tetrahedrally coordinated, which can be described in parallel to a fluorite structure of systematic $\mathrm{Cd} / \mathrm{As}$ vacancies. There are four layers per unit and the missing $\mathrm{Cd}-\mathrm{As}_{4}$ tetrahedra are arranged without the central symmetry as shown with the (001) projection view in Fig. 1b, with the two vacant sites being at diagonally opposite corners of a cube face ${ }^{24}$. The corresponding $\mathrm{BZ}$ is shown in Fig. 1d, where the centre of the BZ is the $\Gamma$ point, the centres of the top and bottom square surfaces are the $Z$ points, and other high-symmetry points are also noted. $\mathrm{Cd}_{3} \mathrm{As}_{2}$ has attracted attention in electrical transport because of its high mobility of $10^{5} \mathrm{~cm}^{2} \mathrm{~V}^{-1} \mathrm{~s}^{-1}$ reported in previous studies 22,23 . The carrier density and mobility of our $\mathrm{Cd}_{3} \mathrm{As}_{2}$ samples (shown in Figs. 1 and 2) are characterized to be of $5.2 \times 10^{18} \mathrm{~cm}^{-3}$ and $42,850 \mathrm{~cm}^{2} \mathrm{~V}^{-1} \mathrm{~s}^{-1}$, respectively, at temperature of $130 \mathrm{~K}$, consistent with previous reports ${ }^{22,23}$, which provide an evidence for the high quality of our single crystalline sample. In band theoretical calculations, $\mathrm{Cd}_{3} \mathrm{As}_{2}$ is also of interest as it features an inverted band structure ${ }^{25}$. More interestingly, a very recent theoretical prediction ${ }^{14,}$ which motivated this work, has shown that the spin-orbit interaction in $\mathrm{Cd}_{3} \mathrm{As}_{2}$ cannot open up a full energy gap between the inverted bulk conduction and valence bands due to the protection of an additional crystallographic symmetry ${ }^{12}$ (in the case of $\mathrm{Cd}_{3} \mathrm{As}_{2}$ it is the $C_{4}$ rotational symmetry along the $k_{z}$ direction ${ }^{14}$ ), which is in contrast to other band-inverted systems such as $\mathrm{HgTe}^{3}$. This theory predicts ${ }^{14}$ that the $C_{4}$ rotational symmetry protects two bulk (3D) Dirac band touching points at two special $\mathbf{k}$ points along the $\Gamma-Z$ momentum space cut-direction, as shown by the red crossings in Fig. 1d. Therefore, $\mathrm{Cd}_{3} \mathrm{As}_{2}$ serves a candidate for a spacegroup or crystal structure symmetry protected $C_{4}$ BDS phase.

Observation of bulk Dirac cone. In order to experimentally identify such a BDS phase, we systematically study the electronic structure of $\mathrm{Cd}_{3} \mathrm{As}_{2}$ on the cleaved (001) surface. Figure 1c shows momentum-integrated ARPES spectral intensity over a wide energy window. Sharp ARPES intensity peaks at binding energies of $E_{\mathrm{B}} \simeq 11 \mathrm{eV}$ and $41 \mathrm{eV}$ that correspond to the cadmium $4 d$ and the arsenic $3 d$ core levels are observed, confirming the chemical composition of our samples. We study the overall electronic structure of the valence band. Figure le shows the second derivative image of an ARPES dispersion map in a 3-eV binding energy window, where the dispersion of several valence bands are identified. Moreover, a low-lying small feature that crosses the Fermi level is observed. In order to resolve it, high-resolution ARPES dispersion measurements are performed in the close vicinity of the Fermi level as shown in Fig. 1f. Remarkably, a linearly dispersive upper Dirac cone is observed at the surface BZ centre $\bar{\Gamma}$ point, whose Dirac node is found to locate at a binding energy of $E_{\mathrm{B}} \simeq 0.2 \mathrm{eV}$. At the Fermi level, only the upper Dirac band but no other electronic states are observed. On the other hand, the linearly dispersive lower Dirac cone is found to coexist with another parabolic bulk valence band, which can be seen from Fig. 1e. From the observed steep Dirac dispersion (Fig. 1f), we obtain a surprisingly high Fermi velocity of about $9.8 \mathrm{eVÅ}$; $\left(\simeq 1.5 \times 10^{6} \mathrm{~ms}^{-1}\right)$. This is more than tenfold larger than the theoretical prediction of $0.15 \mathrm{eVA}$; at the corresponding location of the chemical potential ${ }^{14}$. Compared with the much-studied 2D Dirac systems, the Fermi velocity of the 3D Dirac fermions in $\mathrm{Cd}_{3} \mathrm{As}_{2}$ is thus about 3 times higher than that of in the topological surface states of $\mathrm{Bi}_{2} \mathrm{Se}_{3}$ (ref. 6), 1.5 times higher than in graphene ${ }^{26}$ and 30 times higher than that in the topological Kondo insulator phase in $\mathrm{SmB}_{6}$ (refs 27,28). The observed large Fermi velocity of the 3D Dirac band provides clues to understand unusually high mobility of $\mathrm{Cd}_{3} \mathrm{As}_{2}$ reported in previous transport experiments ${ }^{22,23}$. Therefore, one can expect to observe unusual magneto-electrical and quantum Hall transport properties under high-magnetic field. It is well-known that in graphene the 
a

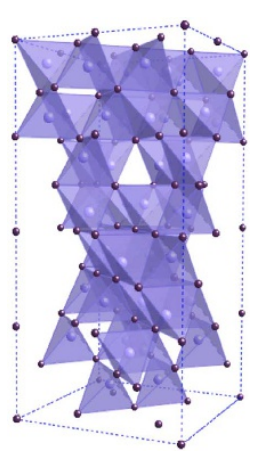

d

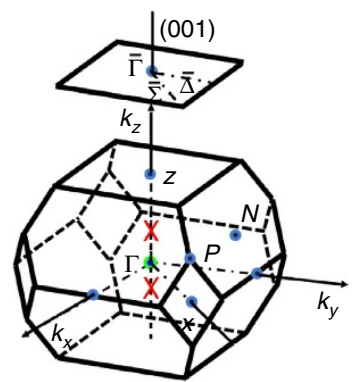

b

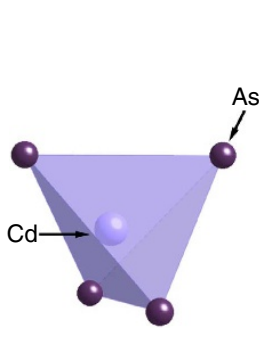

c

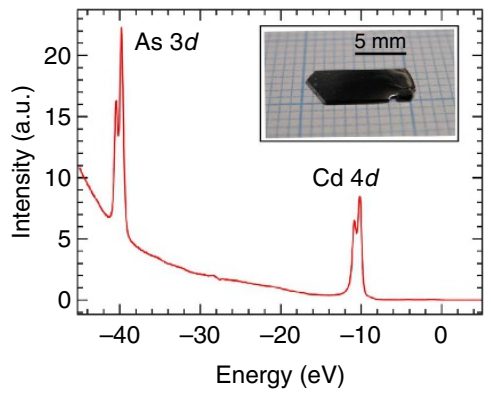

e

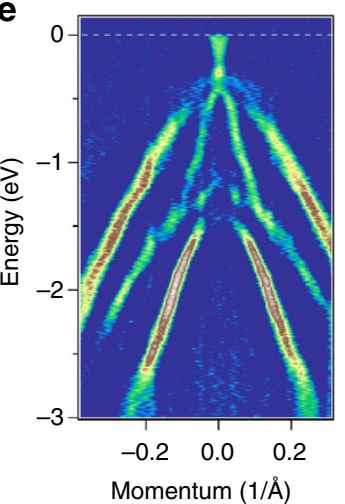

$f$

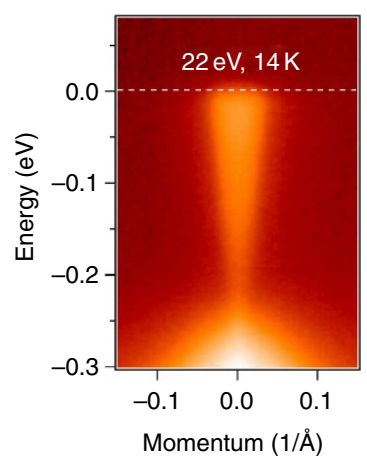

Figure 1 | Brillouin zone symmetry and 3D Dirac cone. (a) $\mathrm{Cd}_{3} \mathrm{As}_{2}$ crystalizes in a tetragonal body centre structure with space group of $/ 4_{1} c d$, which has 32 number of formula units in the unit cell. The tetragonal structure has lattice constant of $a=12.670 \AA, b=12.670 \AA$ and $c=25.480 \AA$. (b) The basic structure unit is a four corner-sharing $\mathrm{CdAs}_{3}$-trigonal pyramid. (c) Core-level spectroscopic measurement where $\mathrm{Cd} 4 d$ and As $3 d$ peaks are clearly observed. Inset shows a picture of the $\mathrm{Cd}_{3} \mathrm{As}_{2}$ samples used for angle-resolved photoemission spectroscopy (ARPES) measurements. The flat and mirrorlike surface indicate the high quality of our samples. (d) The bulk BZ and the projected surface BZ along the (001) direction. The red crossings locate at $\left(k_{\mathrm{x}}, k_{\mathrm{y}}, k_{\mathrm{z}}\right)=\left(0,0,0.15(2 \pi) /\left(c^{\star}\right)\right)\left(c^{\star}=c / a\right)$. They denote the two special $\mathbf{k}$ points along the $\Gamma-Z$ momentum space cut-direction, where $3 D$ Dirac bandtouchings are protected by the crystalline $C_{4}$ symmetry along the $k_{z}$ axis. (e) Second derivative image of ARPES dispersion map of $C d_{3} A s_{2}$ over the wider binding energy range. Various bands are well-resolved up to $3 \mathrm{eV}$ binding energy range. (f) ARPES $E_{\mathrm{B}}-k_{\mathrm{x}}$ cut of $\mathrm{Cd}_{3} \mathrm{As}_{2}$ near the Fermi level at around surface BZ centre $\bar{\Gamma}$ point.

capability to prepare high-quality and high-mobility samples has enabled the experimental observations of many interesting phenomena that arises from its 2D Dirac fermions. The large Fermi velocity and high mobility in $\mathrm{Cd}_{3} \mathrm{As}_{2}$ are among the important experimental criteria to explore the $3 \mathrm{D}$ relativistic physics in various Hall phenomena in tailored $\mathrm{Cd}_{3} \mathrm{As}_{2}$.

We compare ARPES observations with our theoretical calculations, which is qualitatively consistent with previous calculations ${ }^{14}$. The reason for the use of our calculations is twofold: first, our calculations are fine tuned based on the characterization of samples used in the present ARPES study; second, sufficiently detailed cuts are not readily available from ref. 14 , which is necessary for a detailed comparison of ARPES data with theory. In theory, there are two 3D Dirac nodes that are expected at two special $\mathbf{k}$ points along the $\Gamma-Z$ momentum space cut-direction, as shown by the red crossings in Fig. 1d. At the (001) surface, these two $\mathbf{k}$ points along the $\Gamma-Z$ axis project on to the $\bar{\Gamma}$ point of the (001) surface BZ (Fig. 1d). Therefore, at the (001) surface, theory predicts one $3 \mathrm{D}$ Dirac cone at the BZ centre $\bar{\Gamma}$ point, as shown in Fig. 2a. These results are in qualitative agreement with our data, which supports our experimental observation of the $3 \mathrm{D}$ BDS phase in $\mathrm{Cd}_{3} \mathrm{As}_{2}$. We also study the ARPES measured constant energy contour maps (Fig. 2c and d). At the Fermi level, the constant energy contour consists of a single pocket centred at the $\bar{\Gamma}$ point. With increasing binding energy, the size of the pocket decreases and eventually shrinks to a point (the $3 \mathrm{D}$ Dirac point) near $E_{\mathrm{B}} \simeq 0.2 \mathrm{eV}$. The observed anisotropies in the iso-energetic contours are likely due to matrix element effects associated with the standard $p$-polarization geometry used in our measurements.

3D dispersive nature. A $3 \mathrm{D}$ Dirac semimetal is expected to feature nearly linear dispersion along all three momentum space directions close to the crossing point, even though the Fermi/ Dirac velocity can vary significantly along different directions. It is well known that in real materials, such as pure $\mathrm{Bi}$, graphene or TIs, the Dirac cones are never perfectly linear over a large energy window yet they can be approximated to be so within a narrow energy window and in comparison to the large effective mass of conventional band electrons in many other materials. In order to probe the $3 \mathrm{D}$ nature of the observed low-energy Dirac-like bands in $\mathrm{Cd}_{3} \mathrm{As}_{2}$, we performed ARPES measurements as a function of incident photon energy to study the out-of-plane dispersion perpendicular to the (001) surface. Upon varying the photon energy, one can effectively probe the electronic structure at different out-of-plane momentum $k_{z}$ values in a $3 \mathrm{D} \mathrm{BZ}$ and compare with band calculations. In $\mathrm{Cd}_{3} \mathrm{As}_{2}$, the electronic structure or band dispersions in the vicinity of its 3D Dirac-like node can be approximated as: $v_{\|}^{2}\left(k_{x}^{2}+k_{y}^{2}\right)+v_{\perp}^{2}\left(k_{z}-k_{0}\right)^{2}=E^{2}$, where $k_{0}$ is the out-of-plane momentum value of the 3D Dirac point. Thus, at a fixed $k_{z}$ value (which is determined by the incident photon energy value), the in-plane electronic dispersion takes the form: $v_{\|}^{2}\left(k_{x}^{2}+k_{y}^{2}\right)=E^{2}-v_{\perp}^{2}\left(k_{z}-k_{0}\right)^{2}$. It can be seen that only at $k_{z}=k_{0}$ the in-plane dispersion is a gapless Dirac cone, whereas in the case for $k_{z} \neq k_{0}$ the nonzero $k_{z}-k_{0}$ term acts as an 
a

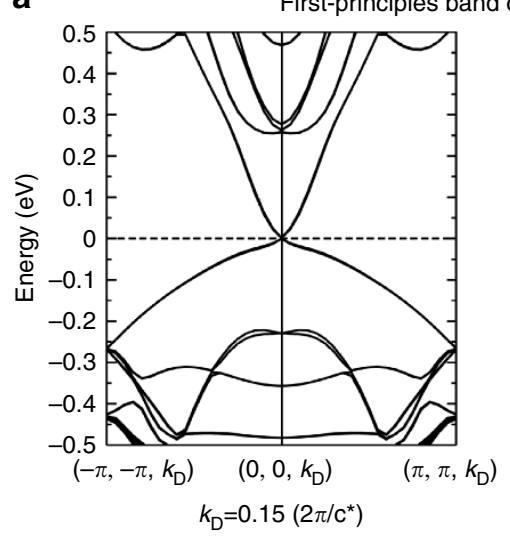

b

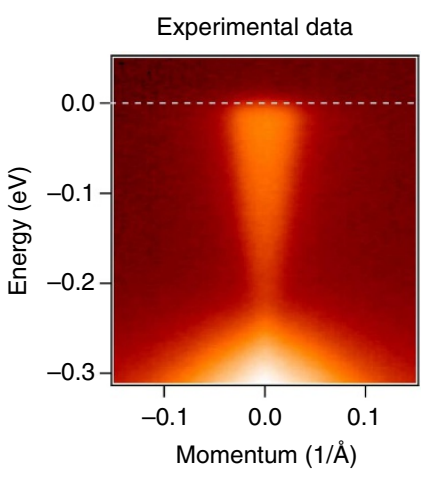

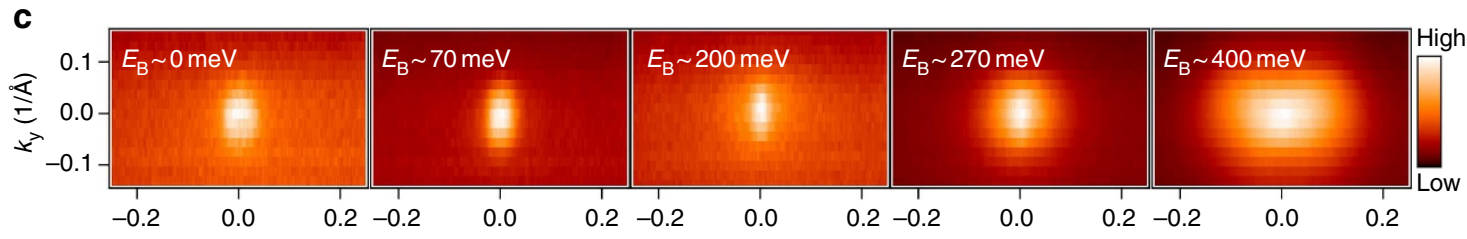

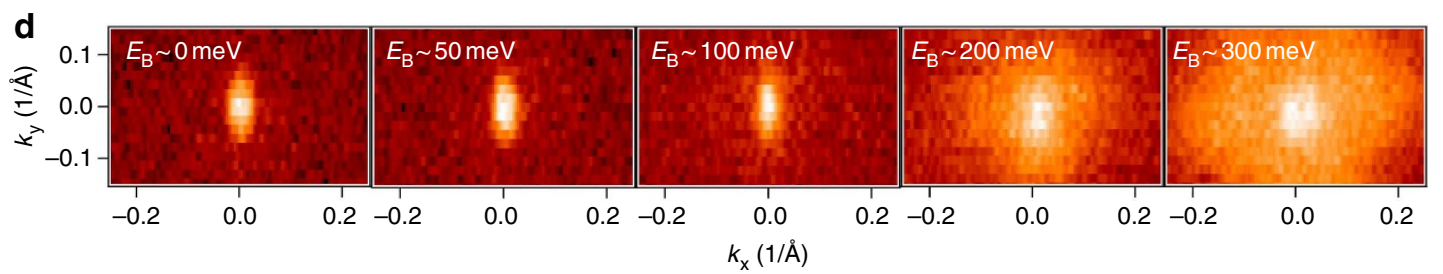

Figure 2 | Observation of in-plane dispersion in $\mathbf{C d}_{\mathbf{3}} \mathbf{A s}_{\mathbf{2}}$. (a) Left: First-principles calculation of the bulk electronic structure along the $(\pi, \pi, 0.15(2 \pi) /$ $\left.\left(c^{\star}\right)\right)-\left(0,0,0.15(2 \pi) /\left(c^{\star}\right)\right)$ direction $\left(c^{\star}=c / a\right)$. Right: Projected bulk band structure on to the (001) surface, where the shaded area shows the projection of the bulk bands. (b) Angle-resolved photoemission spectroscopy (ARPES) measured dispersion map of $\mathrm{Cd}_{3} \mathrm{As}_{2}$, measured with photon energy of $22 \mathrm{eV}$ and temperature of $15 \mathrm{~K}$ along the $(-\pi,-\pi)-(0,0)-(\pi, \pi)$ momentum space cut direction. (c) ARPES constant energy contour maps using photon energy of $22 \mathrm{eV}$ on $\mathrm{Cd}_{3} \mathrm{As}_{2}$ growth batch I for binding energies $0 \mathrm{meV}, 70 \mathrm{meV}, 200 \mathrm{meV}, 270 \mathrm{meV}$ and 400 meV for panel from left to right, respectively. (d) ARPES constant energy contour maps using photon energy of $102 \mathrm{eV}$ on $\mathrm{Cd}_{3} \mathrm{As}_{2}$ batch II for binding energies 0 meV, $50 \mathrm{meV}, 100 \mathrm{meV}, 200 \mathrm{meV}$ and $300 \mathrm{meV}$ for panel from left to right, respectively. In order to achieve chemical potential (carrier concentration) control, we have prepared different batches of samples under slightly different growth conditions (temperature and growth time). For the two batches studied here, batch I is found to be slightly more $n$-type than batch II (e.g. compare batch I in Fig. If with batch II in Fig. 3a rightmost panel).

effective mass term and opens up a gap in the in-plane dispersion relation. Figure $3 \mathrm{a}$ shows the ARPES measured in-plane electronic dispersion at various photon energies. At a photon energy of $102 \mathrm{eV}$, a gapless Dirac-like cone is observed, which shows that photon energy $h v=102 \mathrm{eV}$ corresponds to a $k_{z}$ value that is close to the out-of-plane momentum value of the 3D Dirac node $k_{0}$. As photon energy is changed away from $102 \mathrm{eV}$ in either direction, the bulk conduction and valence bands are observed within experimental resolution to be separated along the energy axis and a gap opens in the in-plane dispersion. At photon energies sufficiently away from $102 \mathrm{eV}$, such as $90 \mathrm{eV}$ or $114 \mathrm{eV}$ in Fig. 3a, the in-plane gap is large enough so that the bottom of the upper Dirac cone (bulk conduction band) is moved above the Fermi level, and therefore only the lower Dirac cone is observed. We now fix the in-plane momenta at 0 and plot the ARPES data at $k_{x}=k_{y}=0$ as a function of incidence photon energy. As shown in Fig. $3 \mathrm{~b}, \mathrm{a} E-k_{z}$ dispersion is observed in the out-of-plane momentum space cut direction, which is in qualitative agreement with the theoretical calculations (Fig. 3c). The Fermi velocity in the $z$-direction can be estimated (only at the order of magnitude level) to be about $10^{5} \mathrm{~ms}^{-1}$. We note that the sample we used for $k_{z}$ dispersion measurements (Fig. $3 \mathrm{a}-\mathrm{c}$ ) is relatively $p$-type (Fermi velocity is about $80 \mathrm{meV}$ from the Dirac point) as compared with the sample we used to measure the in-plane dispersion and Fermi surfaces (Figs 1 and 2) where chemical potential is about $200 \mathrm{meV}$ from the Dirac point. It is important to note that the magnitude of Fermi velocity anisotropy strongly depends on the position of the sample chemical potential ( $n$-type sample leads to weaker anisotropy), and therefore the direct comparison between our results and previous transport data in terms of this anisotropy is not applicable. These systematic incident photon energy-dependent measurements show that the observed Dirac-like band disperses along both the in-plane and the out-of-plane directions, suggesting its $3 \mathrm{D}$ or bulk nature consistent with theory.

In order to further understand the nature of the observed Dirac band, we studied the spin polarization or spin texture properties of $\mathrm{Cd}_{3} \mathrm{As}_{2}$. As shown in Fig. 3f, spin-resolved ARPES measurements are performed on a relatively $p$-type sample. Two spin-resolved energy-dispersive curve cuts are shown at momenta of $\pm 0.1 \AA^{-1}$ on the opposite sides of the Fermi surface. The obtained spin data shown in Fig. 3g,h show no observable net spin polarization or texture behaviour within our experimental resolution, which is in remarkable contrast with the clear spin texture in 2D Dirac fermions on the surfaces of TIs. The absence of spin texture in our observed Dirac fermion in $\mathrm{Cd}_{3} \mathrm{As}_{2}$ bands is consistent with their bulk origin, which agrees with the theoretical prediction. It also provides a strong evidence that our ARPES signal is mainly due to the bulk Dirac bands on the surface of $\mathrm{Cd}_{3} \mathrm{As}_{2}$, whereas the 
a
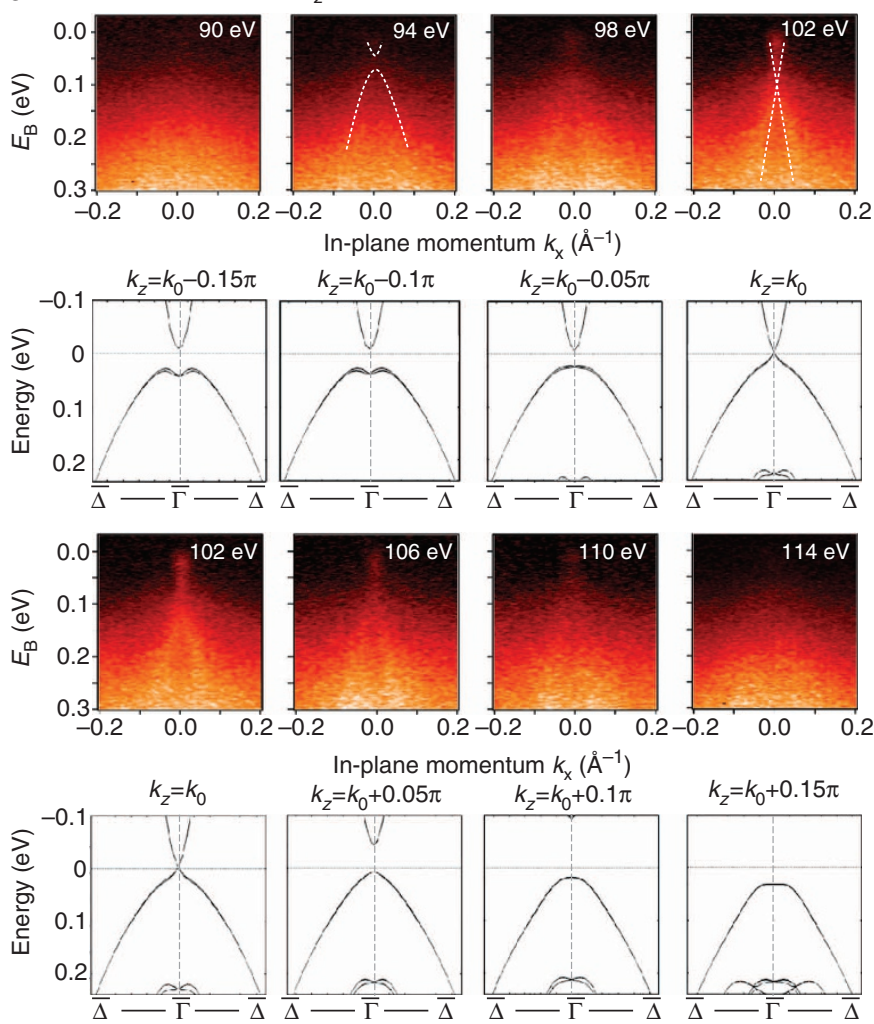

In-plane momentum $k_{\mathrm{x}}\left(\AA^{-1}\right)$
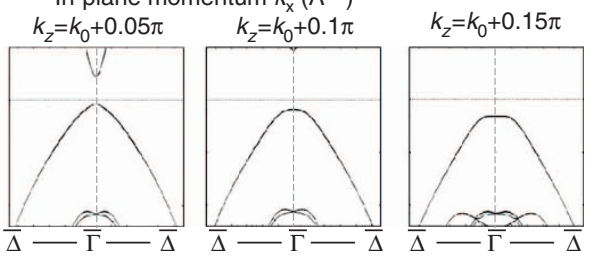

g

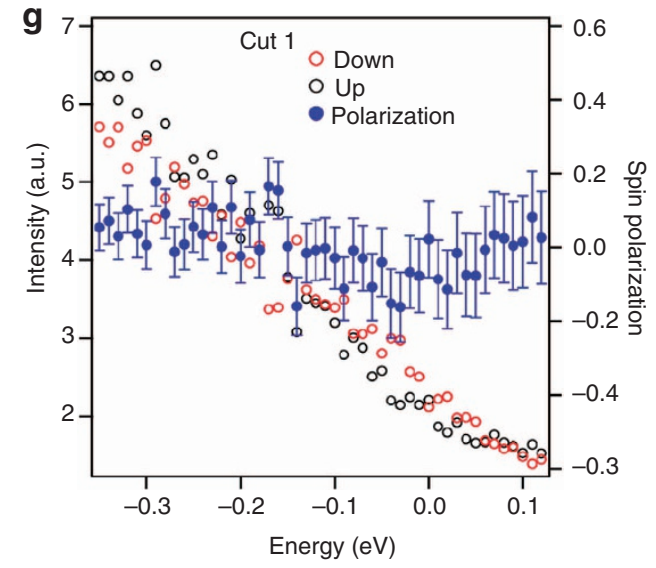

b
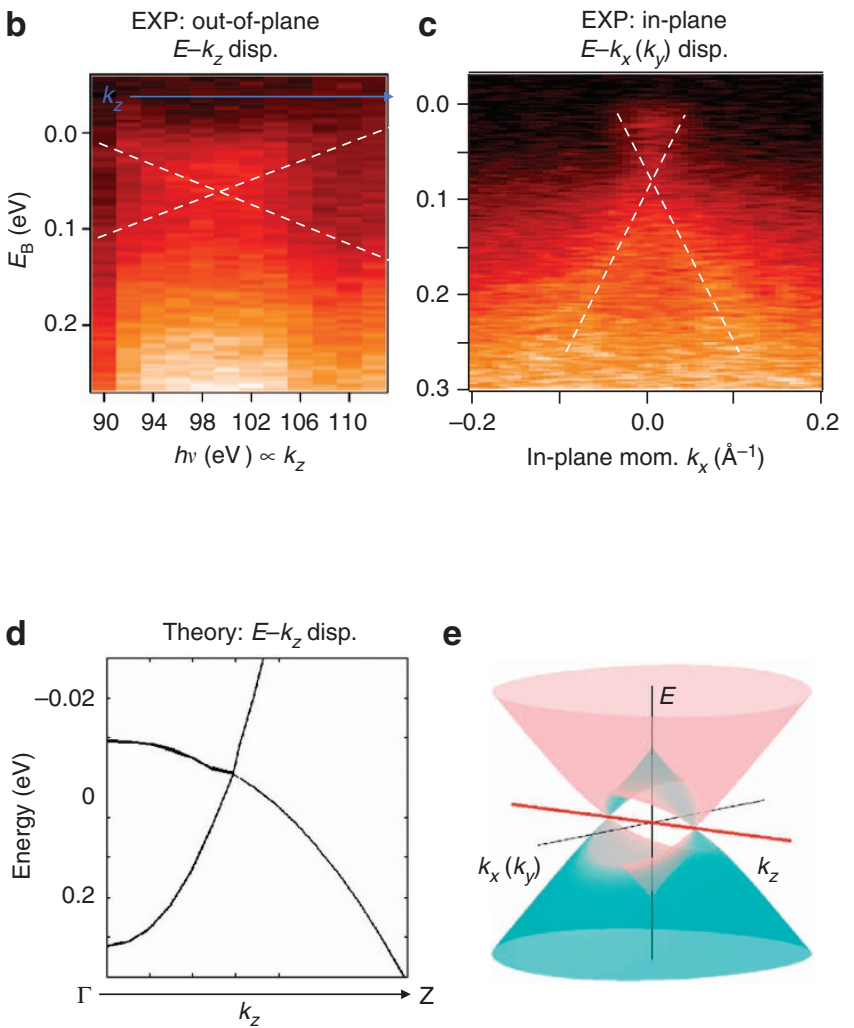

e

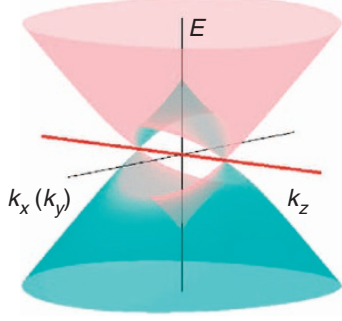

f

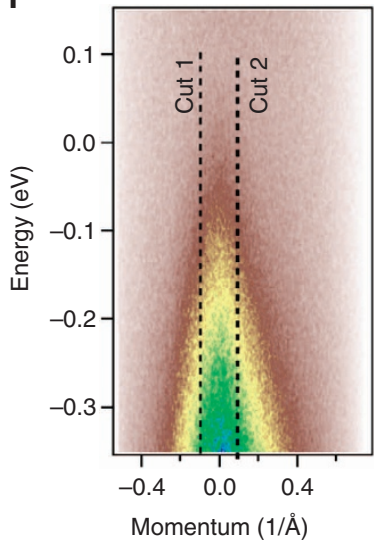

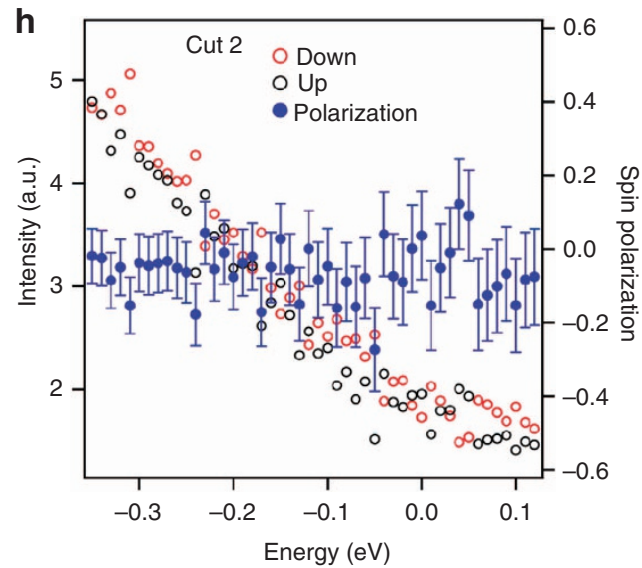

Figure 3 | Observation of out-of-plane dispersion in $\mathbf{C d}_{\mathbf{3}} \mathbf{A s}_{\mathbf{2}}$. (a) Angle-resolved photoemission spectroscopy (ARPES) dispersion maps at various incident photon energies are shown in the first and third rows. First-principle calculated in-plane electronic dispersion at different $k_{z}$ values near the three-dimensional (3D) Dirac node $k_{0}$ is plotted in the second and forth rows. (b) ARPES measured out-of-plane linear $E-k_{z}$ dispersion. (c) ARPES measured in-plane $E-k_{x}$ dispersion. The white dotted lines are guides to the eye tracking the out-of-plane dispersion. (d) Theoretically calculated out-of-plane $E-k_{z}$ dispersion near the 3D Dirac node shown over a wider energy window. (e) Schematic (cartoon) of the 3D (anisotropic) Dirac semimetal band structure in $\mathrm{Cd}_{3} \mathrm{As}_{2}$. (f) Spin-integrated ARPES dispersion cut measured on the sample used for spin-resolved measurements. The dotted lines indicate the momentum locations for the spin-resolved energy-dispersive curve cuts. ( $\mathbf{g}$ and $\mathbf{h}$ ) Spin-resolved ARPES intensity (black and red circles) and measured net spin polarization (blue dots) for cuts 1 and 2. Error bars represent the experimental uncertainties (standard deviation) in determining the spin polarization.

predicted surface (resonance) states ${ }^{14}$ that lie along the boundary of the bulk Dirac cone projection has a small spectral weight (intensity) contribution to the photoemission signal. In other words, according to our experimental data, the surface electronic structure of $\mathrm{Cd}_{3} \mathrm{As}_{2}$ is dominated by the spin-degenerate bulk bands, which is very different from that of the $3 \mathrm{D}$ TIs.

\section{Discussion}

The distinct semimetal nature of $\mathrm{Cd}_{3} \mathrm{As}_{2}$ is better understood from ARPES data if we compare our results with that of the prototype $\mathrm{TI}, \mathrm{Bi}_{2} \mathrm{Se}_{3}$. In $\mathrm{Bi}_{2} \mathrm{Se}_{3}$ as shown in Fig. $4 \mathrm{~b}$, the bulk conduction and valence bands are fully separated (gapped), and a linearly dispersive topological surface state is observed that connect across the bulk band-gap. In the case of $\mathrm{Cd}_{3} \mathrm{As}_{2}$ (Fig. $4 \mathrm{a}$ ), there does not exist a full bulk energy gap. On the other hand, the bulk conduction and valence bands 'touch' (and only 'touch') at one specific location in the momentum space, which is the $3 \mathrm{D}$ band-touching node, thus realizing a 3D BDS. For comparison, we further show that a similar BDS state is also realized by tuning the chemical composition $\delta$ (effectively the spin-orbit coupling 
a
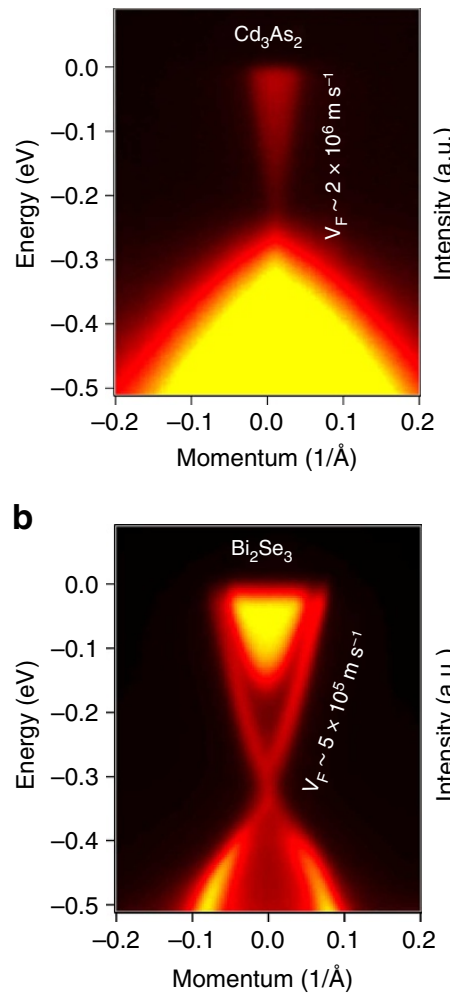
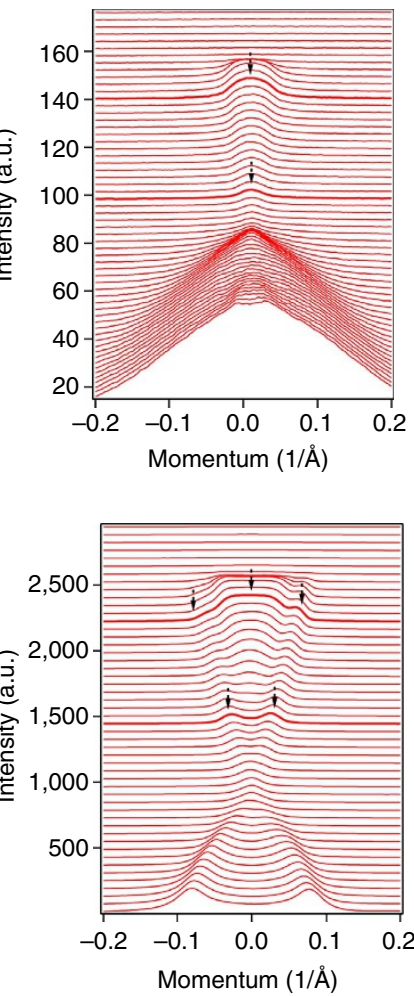
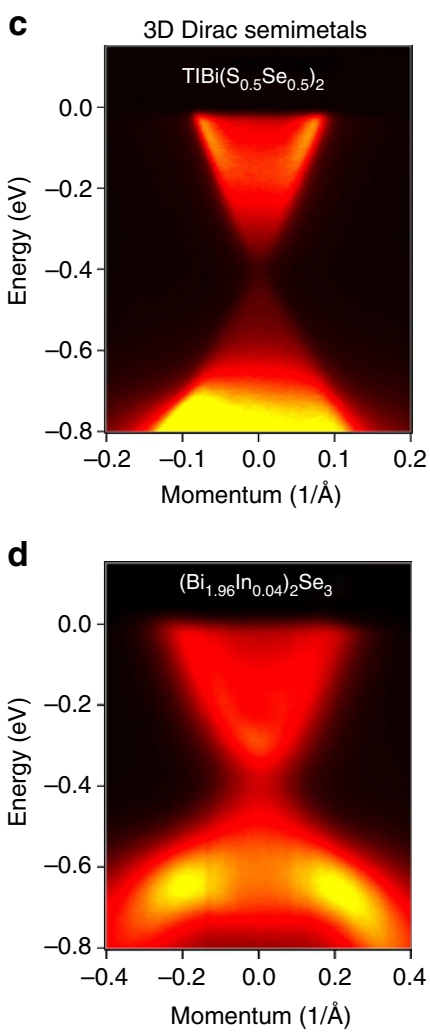

Figure 4 | Two- and three-dimensional topological Dirac fermions. (a) Angle-resolved photoemission spectroscopy (ARPES) measured surface electronic structure dispersion map of $\mathrm{Cd}_{3} \mathrm{As}_{2}$ and its corresponding momentum distribution curves (MDCs). (b) ARPES measured surface dispersion map of the prototype topological insulator $(\mathrm{TI}) \mathrm{Bi}_{2} \mathrm{Se}_{3}$ and its corresponding MDCs. Both spectra are measured with photon energy of 22 eV and at a sample temperature of $15 \mathrm{~K}$. The black arrows show the ARPES intensity peaks in the MDC plots. (c and d) ARPES spectra of two Bi-based 3D Dirac semimetals, which are realized by fine tuning the chemical composition to the critical point of a topological phase transition between a normal insulator and a TI: (c) $\mathrm{TIBi}\left(\mathrm{S}_{1-\delta} \mathrm{Se}_{\delta}\right)_{2}\left(\delta=0.5\right.$; see Xu et al. $\left.{ }^{20}\right)$, and (d) $\left(\mathrm{Bi}_{1}-{ }_{\delta} \mathrm{ln}_{\delta}\right)_{2} \mathrm{Se}_{3}\left(\delta=0.04\right.$; see Brahlek et al. $\left.{ }^{29}\right)$. Spectrum in c is measured with photon energy of 16 eV and spectrum in $\mathbf{d}$ is measured with photon energy of $41 \mathrm{eV}$. For the $2 \mathrm{D}$ topological surface Dirac cone in $\mathrm{Bi}_{2} \mathrm{Se}_{3}$, a distinct in-plane ( $\left.E_{\mathrm{B}}-k_{x}\right)$ dispersion is observed in ARPES, whereas for the $3 \mathrm{D}$ bulk Dirac cones in $\mathrm{Cd}_{3} \mathrm{As}_{2}$, $\mathrm{TIBi}\left(\mathrm{S}_{0.5} \mathrm{Se}_{0.5}\right)_{2}$ and $\left(\mathrm{Bi}_{0.96} \mathrm{In}_{0.04}\right)_{2} \mathrm{Se}_{3}$, a Dirac-cone-like intensity continuum is also observed.

strength) to the critical point of a topological phase transition between a normal insulator and a TI. Figure $4 \mathrm{c}, \mathrm{d}$ presents the surface electronic structure of two other BDS phases in the $\operatorname{BiTl}\left(\mathrm{S}_{1-\delta} \mathrm{Se}_{\delta}\right)_{2}$ and $\left(\mathrm{Bi}_{1-\delta} \operatorname{In}_{\delta}\right)_{2} \mathrm{Se}_{3}$ systems. In both systems, it has been shown that tuning the chemical composition $\delta$ can drive the system from a normal insulator state to a TI state $20,21,29$. The critical compositions for the two topological phase transitions are approximately near $\delta=0.5$ and $\delta=0.04$, respectively. Figure 4c,d shows the ARPES measured surface electronic structure of the critical compositions for both $\operatorname{BiTl}\left(\mathrm{S}_{1-\delta} \mathrm{Se}_{\delta}\right)_{2}$ and $\left(\mathrm{Bi}_{1-\delta} \mathrm{In}_{\delta}\right)_{2} \mathrm{Se}_{3}$ systems, which are expected to exhibit the BDS phase. Indeed, the bulk critical compositions where bulk and surface Dirac bands collapse also show Dirac cones with intensities filled inside the cones, which is qualitatively similar to the case in $\mathrm{Cd}_{3} \mathrm{As}_{2}$. Currently, the origin of the filling behaviour is not fully understood irrespective of the bulk (out-of-plane dispersive behaviour) nature of the overall band dispersion interpreted in connection to band calculations (see Fig. 2). Based on the ARPES data in Fig. 4c,d, the Fermi velocity is estimated to be $\sim 4 \mathrm{eV} \AA$ and $\sim 2 \mathrm{eV} \AA$ for the 3D Dirac fermions in $\operatorname{BiTl}\left(\mathrm{S}_{1}{ }_{\delta} \mathrm{Se}_{\delta}\right)_{2}$ and $\left(\mathrm{Bi}_{1-\delta} \operatorname{In}_{\delta}\right)_{2} \mathrm{Se}_{3}$, respectively, which is much lower than that of what we observe in $\mathrm{Cd}_{3} \mathrm{As}_{2}$, thus likely limiting the carrier mobility. The mobility is also limited by the disorder due to the strong chemical alloying. More importantly, the fine control of doping/alloying $\delta$ value and keeping the composition exactly at the bulk critical composition is difficult to achieve $e^{20}$, especially while considering the chemical inhomogeneity introduced by the dopants. For example, although similarly high electron mobility on the order of $10^{5} \mathrm{~cm}^{2} \mathrm{~V}^{-1} \mathrm{~s}^{-1}$ has been reported in the bulk states of $\mathrm{Pb}_{1-x} \mathrm{Sn}_{x} \mathrm{Se}(x=0.23)$ (ref. 30$)$, the bulk Dirac fermions are in fact massive because of the difficulty of controlling the composition exactly at the critical point. These facts taken together exclude the possibility of realizing proposed topological physics including the Weyl semimetal and quantum spin Hall phases using the bulk Dirac states in the $\mathrm{Pb}_{1-x} \mathrm{Sn}_{x} \mathrm{Se}$. These issues do not arise in the stoichiometric $\mathrm{Cd}_{3} \mathrm{As}_{2}$ system as its BDS phase is protected by the crystal symmetry, which does not require chemical doping and therefore the natural high electron mobility is retained (not diminished). We note that our crystals of $\mathrm{Cd}_{3} \mathrm{As}_{2}$ are nearly stoichiometric within the resolution of electron probe micro-analyser (EPMA) and X-ray diffraction analysis. The existence of some low-level defects is not ruled out. However, these defects do not affect the main conclusion regarding the $3 \mathrm{D}$ Dirac band structure ground state of this compound. Beside $\mathrm{Cd}_{3} \mathrm{As}_{2}$ and the topological phase transition critical composition samples as discussed above, we also note that BDSs unrelated to the combination of $C_{4}$ symmetry and band-inverted spin-orbit coupling (combination of which has been termed 'topological' in theory ${ }^{14}$ ) have been studied previously in pnictide $\mathrm{BaFe}_{2} \mathrm{As}_{2}$ (ref. 31), heavy fermion $\mathrm{LaRhIn}_{5}$ (ref. 32) and organic compound $\alpha$-(BEDT-TTF $)_{2} \mathrm{I}_{3}$ (ref. 33). The recent interest is actually focused on spin-orbit-based 3D BDS phase as the spin-orbit coupling can 
drive exotic topological phenomena and quantum transport in such materials as the Weyl phases, high-temperature linear quantum magnetoresistance and topological magnetic phases $^{11-14,16-19}$. Our observation of the bulk Dirac states in $\mathrm{Cd}_{3} \mathrm{As}_{2}$ provides a unique combination of physical properties, including high spin-orbit coupling strength, high electron mobility, massless nature guaranteed by the crystal symmetry protection without compositional tuning, making it an ideal and unique platform to realize many of the proposed exciting new topological physics ${ }^{11-14,16-19}$.

In conclusion, we have experimentally identified the crystalline-symmetry-protected 3D spin-orbit BDS phase in a stoichiometric system $\mathrm{Cd}_{3} \mathrm{As}_{2}$ (see Fig. 5). The combination of a large Fermi velocity and very high electron mobility of the $3 \mathrm{D}$ carriers with nearly linear dispersion at the crossing point makes it a promising platform to explore novel $3 \mathrm{D}$ relativistic physics in various types of quantum Hall phenomena. Our band structure study of the predicted 3D BDS phase also paves the way for designing and realizing a number of related exotic topological phenomena in future experiments. For example, if the $C_{4}$ crystalline symmetry is broken, the $3 \mathrm{D}$ Dirac cone in $\mathrm{Cd}_{3} \mathrm{As}_{2}$ can open up a gap and therefore a TI phase is realized in a highmobility setting (current Bi-based TIs feature low carrier mobility). Furthermore, upon doping magnetic elements or fabricating superlattice hetero-structures, the $3 \mathrm{D}$ Dirac node in $\mathrm{Cd}_{3} \mathrm{As}_{2}$ can be split into two topologically protected Weyl nodes, realizing the much sought out Fermi arcs phases in solid-state setting.
During the preparation of this manuscript, we became aware of another study ${ }^{34}$ reporting ARPES studies of experimental realization of $3 \mathrm{D}$ Dirac semimetal phase in $\mathrm{Cd}_{3} \mathrm{As}_{2}$; however, many of the experimental details and interpretations of the data differ from ours. Two other studies $^{35,36}$ also reported experimental realization of the $3 \mathrm{D}$ Dirac phase in a metastable low-mobility compound, $\mathrm{Na}_{3} \mathrm{Bi}$.

\section{Methods}

Sample growth and characterization. Single crystalline samples of $\mathrm{Cd}_{3} \mathrm{As}_{2}$ were grown using the standard method, which is described elsewhere ${ }^{24}$. The $\mathrm{Cd}_{3} \mathrm{As}_{2}$ samples used for our ARPES studies show carrier density of $5.2 \times 10^{18} \mathrm{~cm}^{-3}$ and mobility up to $42,850 \mathrm{~cm}^{2} \mathrm{~V}^{-1} \mathrm{~s}^{-1}$ at temperature of $130 \mathrm{~K}$, which is consistent with the mobility of $10^{4}-10^{5} \mathrm{~cm}^{2} \mathrm{~V}^{-1} \mathrm{~s}^{-1}$ reported elsewhere ${ }^{22,23}$. A slight variation of the value of carrier density and mobility is observed for different growth batch samples. We note that our samples show different chemical potential position (measured by ARPES) and different carrier density (measured by transport) depending on the detailed growth conditions. Moreover, our crystals of $\mathrm{Cd}_{3} \mathrm{As}_{2}$ are nearly stoichiometric within the resolution of electron probe micro-analyser and X-ray diffraction analysis. The existence of some low-level defects is not ruled out.

Spectroscopic measurements. ARPES measurements for the low-energy electronic structure were performed at the PGM beamline in Synchrotron Radiation Center (SRC) in Wisconsin, and at the beamlines 4.0.3, 10.0.1 and 12.0.1 at the Advanced Light Source in Berkeley, California, equipped with high-efficiency VG-Scienta R4000 or R8000 electron analysers. Spin-resolved ARPES measurements were performed at the ESPRESSO endstation at HiSOR. Photoelectrons are excited by an unpolarized $\mathrm{He}-\mathrm{I} \alpha$ light $(21.21 \mathrm{eV})$. The spin polarization is detected by state-of-the-art very-low-energy electron diffraction spin detectors using preoxidized $\mathrm{Fe}(001)-\mathrm{p}(1 \times 1)-\mathrm{O}$ targets ${ }^{37}$. The two spin detectors are placed at an

\section{a}

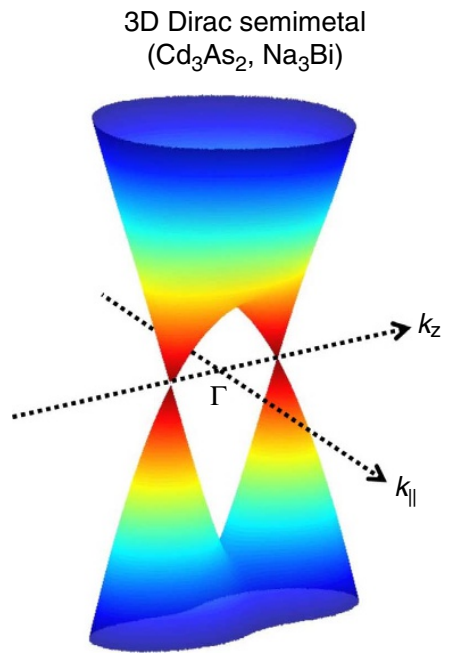

Top view of lower cone

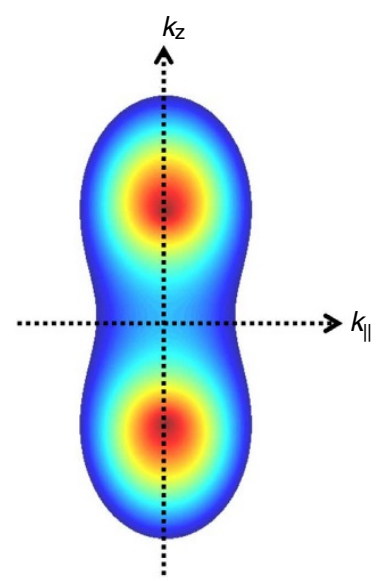

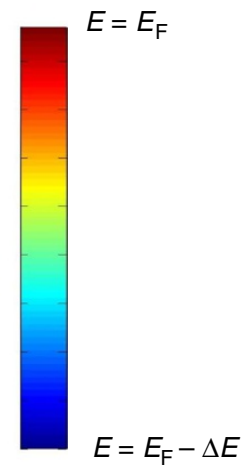

b Fermi surface
$E=E_{\mathrm{D}}$

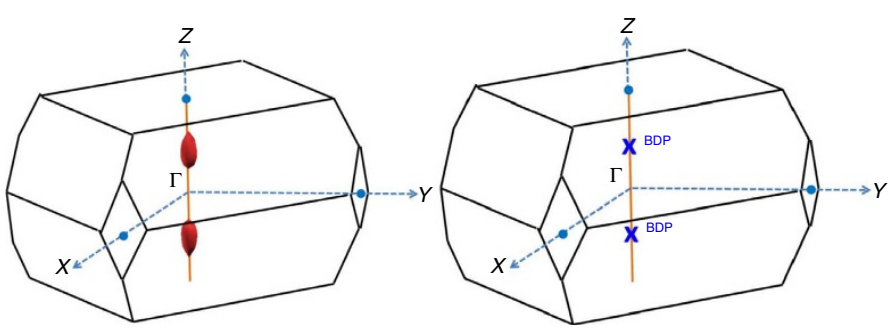

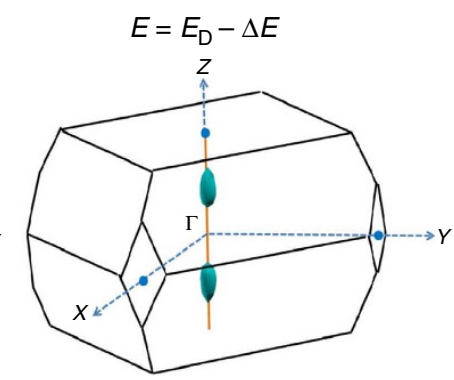

Figure 5 | Three-dimensional Dirac semimetal phase. (a) Cartoon view of dispersion of 3D Dirac semimetal. (b) Schematic view of the Fermi surface above the Dirac point (left panel), at the Dirac point (middle panel) and below the Dirac point (right panel). 
angle of $90^{\circ}$ and are directly attached to a VG-Scienta R4000 hemispheric analyser, enabling simultaneous spin-resolved ARPES measurements for all three spin components as well as high-resolution spin-integrated ARPES experiments. The energy and momentum resolution were better than $40 \mathrm{meV}$ and $1 \%$ of the surface BZ for spin-integrated ARPES measurements at the SRC and the Advanced Light Source, and $80 \mathrm{meV}$ and $3 \%$ of the surface BZ for spin-resolved ARPES measurements at ESPRESSO endstation at HiSOR. Samples were cleaved in situ and measured at $10-80 \mathrm{~K}$ in a vacuum better than $1 \times 10^{-10}$ torr. They were found to be very stable and without degradation for the typical measurement period of $20 \mathrm{~h}$

Theoretical calculations. The first-principles calculations are based on the generalized gradient approximation ${ }^{38}$ using the projector augmented wave method $^{39,40}$ as implemented in the VASP package ${ }^{41,42}$. The experimental crystal structure was used ${ }^{24}$. The electronic structure calculations were performed over $4 \times 4 \times 2$ Monkhorst-Pack k-mesh with the spin-orbit coupling included self-consistently.

\section{References}

1. Weyl, H. Elektron und Gravitation I. Physics 56, 330-352 (1929).

2. Geim, A. K. \& Novoselov, K. S. The rise of graphene. Nat. Mater. 6, 183-191 (2007).

3. Hasan, M. Z. \& Kane, C. L. Colloquium: topological insulators. Rev. Mod. Phys. 82, 3045-3067 (2010).

4. Qi, X.-L. \& Zhang, S.-C. Topological insulators and superconductors. Rev. Mod. Phys. 83, 1057-1110 (2011).

5. Hsieh, D. et al. A topological Dirac insulator in a quantum spin Hall phase. Nature 452, 970-974 (2008).

6. Xia, Y. et al. Observation of a large-gap topological-insulator class with a single Dirac cone on the surface. Nat. Phys. 5, 398-402 (2009).

7. Chen, Y. L. et al. Experimental realization of a three-dimensional topological insulator, $\mathrm{Bi}_{2} \mathrm{Te}_{3}$. Science 325, 178-181 (2009).

8. Hsieh, D. et al. Observation of unconventional quantum spin textures in topological insulators. Science 323, 919-922 (2009).

9. Hasan, M. Z. \& Moore, J. E. Three-dimensional topological insulators. Ann. Rev. Cond. Matter Phys. 2, 55-78 (2011).

10. Li, L. et al. Phase transitions of Dirac electrons in Bismuth. Science 321, 547-550 (2008).

11. Murakami, S. Phase transition between the quantum spin Hall and insulator phases in 3D: emergence of a topological gapless phase. New J. Phys. 9, 356 (2007).

12. Young, S. M. et al. Dirac semimetal in three dimensions. Phys. Rev. Lett. 108, 140405 (2012)

13. Wang, Z. et al. Dirac semimetal and topological phase transitions in $\mathrm{A}_{3} \mathrm{Bi}$ $(\mathrm{A}=\mathrm{Na}, \mathrm{K}, \mathrm{Rb})$. Phys. Rev. B 85, 195320 (2012).

14. Wang, Z. et al. Three-dimensional Dirac semimetal and quantum transport in $\mathrm{Cd}_{3} \mathrm{As}_{2}$. Phys. Rev. B 88, 125427 (2013).

15. Neupane, M. et al. Topological surface states and Dirac point tuning in ternary topological insulators. Phys. Rev. B 85, 235406 (2012)

16. Volovik, G. T. Momentum space topology of fermion zero modes brane. JETP Lett. 75, 55 (2002).

17. Fang, Z. et al. The anomalous hall effect and magnetic monopoles in momentum space. Science 302, 92-95 (2003).

18. Wan, X. et al. Topological semimetal and Fermi-arc surface states in the electronic structure of pyrochlore iridates. Phys. Rev. B 83, 205101 (2011)

19. Halasz, G. B. \& Balents, L. Time-reversal invariant realization of the Weyl semimetal phase. Phys. Rev. B. 85, 035103 (2012).

20. Xu, S.-Y. et al. Topological phase transition and texture inversion in a tunable topological insulator. Science 332, 560-564 (2011).

21. Sato, T. et al. Unexpected mass acquisition of Dirac fermions at the quantum phase transition of a topological insulator. Nat. Phys. 7, 840-844 (2011).

22. Jay-Gerin, J.-P. et al. The electron mobility and the static dielectric constant of $\mathrm{Cd}_{3} \mathrm{As}_{2}$ at $4.2 \mathrm{~K}$. Solid State Commun. 21, 771 (1977).

23. Zdanowicz, L. et al. Shubnikov-de Hass effect in amorhous $\mathrm{Cd}_{3} \mathrm{As}_{2}$ in applications of high magnetic fields in semiconductor physics. Lect. Notes Phys. 177, 386 (1983).
24. Steigmann, G. A. \& Goodyear, J. The crystal structure of $\mathrm{Cd}_{3} \mathrm{As}_{2}$. Acta Cryst. B 24, 1062 (1968).

25. Plenkiewicz, B. D. \& Plenkiewicz, P. Inverted band structure of $\mathrm{Cd}_{3} \mathrm{As}_{2}$. Physica Status Solidi(b) 94, K57 (2006).

26. Bostwick, A. et al. Quasiparticle dynamics in graphene. Nat. Phys. 3, 36 (2007).

27. Lu, F. et al. Correlated topological insulators with mixed valence. Phys. Rev. Lett. 110, 096401 (2013).

28. Neupane, M. et al. Surface electronic structure of the topological Kondo-insulator candidate correlated electron system $\mathrm{SmB}_{6}$. Nat. Commun. 4, 2991 (2013).

29. Brahlek, M. et al. Topological-metal to band-insulator transition in $\left(\mathrm{Bi}_{1-x} \mathrm{In}_{x}\right)_{2} \mathrm{Se}_{3}$ thin films. Phys. Rev. Lett. 109, 186403 (2012).

30. Liang, T. et al. Evidence for massive bulk Dirac Fermions in $\mathrm{Pb}_{1-x} \mathrm{Sn}_{x} \mathrm{Se}$ from Nernst and thermopower experiments. Nat. Commun. 4, 2696 (2013).

31. Richard, P. et al. Observation of Dirac cone electronic dispersion in $\mathrm{BaFe}_{2} \mathrm{As}_{2}$. Phys. Rev. Lett. 104, 137001 (2010)

32. Mikitik, G. P. \& Sharlai, Y. V. Berry phase and de Haas-van Alphen effect in LaRhIn $_{5}$. Phys. Rev. Lett. 93, 106403 (2010).

33. Monteverde, M. et al. Coexistence of Dirac and massive carriers in $\alpha$-(BEDT-TTF) ${ }_{2} \mathrm{I}_{3}$ under hydrostatic pressure. Phy. Rev. B 87, 245110 (2013).

34. Borisenko, S. et al. Experimental realization of a three-dimensional Dirac semimetal. Preprint at http://arxiv.org/abs/1309.7978 (2013).

35. Liu, Z. K. et al. Discovery of a three-dimensional topological Dirac semimetal, $\mathrm{Na}_{3} \mathrm{Bi}$. Science 343, 864-867 (2014).

36. Xu, S.-Y. et al. Observation of a bulk 3D Dirac multiplet. Lifshitz transition, and nestled spin states in $\mathrm{Na}_{3} \mathrm{Bi}$. Preprint at http://arxiv.org/abs/1312.7624 (2013).

37. Okuda, T. et al. Efficient spin resolved spectroscopy observation machine at Hiroshima Synchrotron Radiation Center. Rev. Sci. Instrum. 82, 103302 (2011).

38. Perdew, J. P., Burke, K. \& Ernzerhof, M. Generalized gradient approximation made simple. Phys. Rev. Lett. 77, 3865-3868 (1996).

39. Blochl, P. E. Projector augmented-wave method. Phys. Rev. B 50, 17953 (1994)

40. Kresse, G. \& Joubert, D. From ultrasoft pseudopotentials to the projector augmented-wave method. Phys. Rev. B 59, 1758 (1999).

41. Kresse, G. \& Hafner, J. Ab initio molecular dynamics for open-shell transition metals. Phys. Rev. B 48, 13115 (1993).

42. Kress, G. \& Furthmuller, J. Efficient iterative schemes for ab initio total-energy calculations using a plane-wave basis set. Phys. Rev. B 54, 11169 (1996).

\section{Acknowledgements}

The work at Princeton University and Princeton-led synchrotron X-ray-based measurements and the related theory at Northeastern University are supported by the Office of Basic Energy Sciences, US Department of Energy (grants DE-FG-02-05ER46200, AC03-76SF00098 and DE-FG02-07ER46352). We thank J. Denlinger, S.-K. Mo and A. Fedorov for beamline assistance at the DOE supported Advanced Light Source (ALS-LBNL) in Berkeley. We also thank M. Bissen and M. Severson for beamline assistance at SRC, WI. M.Z.H. acknowledges Visiting Scientist support from LBNL, Princeton University, and the A.P. Sloan Foundation.

\section{Author contributions}

M.N. and S.-Y.X. performed the experiments with assistance from N.A., G.B., C.L., I.B. and M.Z.H.; M.N. and M.Z.H. performed data analysis, figure planning and draft preparation; R.S. and F.C. provided the single-crystal samples and performed sample characterization; T.-R.C., H.-T.J., H.L. and A.B. carried out calculations; M.Z.H. was responsible for the conception and the overall direction, planning and integration among different research units.

\section{Additional information}

Competing financial interests: The authors declare no competing financial interests.

Reprints and permission information is available online at http://npg.nature.com/ reprintsandpermissions/

How to cite this article: Neupane, M. et al. Observation of a three-dimensional topological Dirac semimetal phase in high-mobility $\mathrm{Cd}_{3} \mathrm{As}_{2}$. Nat. Commun. 5:3786 doi: $10.1038 /$ ncomms4786 (2014) 